\title{
Discrete uninorms with smooth underlying operators
}

\author{
Daniel Ruiz-Aguilera ${ }^{1}$ Joan Torrens $^{1}$ \\ ${ }^{1}$ Mathematics and Computer Science Department, Universitat de les Illes Balears \\ Carretera de Valldemossa, km 7,5, 07122 Palma, Spain
}

\begin{abstract}
The class of discrete uninorms $U$ such that their underlying t-norm $T$ and t-conorm $S$ are smooth is studied. The different cases combining when $T$ is the minimum or the Eukasiewicz t-norm and $S$ is the maximum or the Eukasiewicz t-conorm, are characterized and the number of discrete uninorms with these underlying operators is given. It is also studied the general case when $T$ and/or $S$ are smooth, but ordinal sums.
\end{abstract}

Keywords: Discrete uninorms, smoothness condition, t-norms, t-conorms

\section{Introduction}

Uninorms are a especial kind of binary aggregation functions that generalize both t-norms and tconorms. They have proved to be useful in many application fields and this have lead to an extensive study of uninorms from the pure theoretical point of view. One of the most interesting topics in this direction deals with the characterization of the different classes of uninorms, mainly uninorms in $\mathcal{U}_{\text {min }}$ and $\mathcal{U}_{\max }$ [7], idempotent uninorms $[1,16,11]$, representable uninorms $[2,7,15]$, uninorms continuous in the open unit square $[8,5]$, compensatory uninorms [4], and even those uninorms with continuous underlying operators [6].

Uninorms are usually defined on the unit interval $[0,1]$, but they can be generalized to other domains. In particular, uninorms defined on a finite chain have been studied by some authors. The interest of operators defined on a finite chain comes from their usefulness in problems where qualitative information is used. When data is qualitative, the fuzzy linguistic approach is a good tool to model the information because then, the qualitative terms used by experts are represented via linguistic variables instead of numerical values. In these cases, linguistic variables are often interpreted to take values on a totally ordered scale like

$$
\begin{aligned}
\mathcal{L}= & \{\text { Extremely Bad, Very Bad, Bad, Fair, Good, } \\
& \text { Very Good, Extremely Good }\}
\end{aligned}
$$

Then the representative finite chain $L_{n}=$ $\{0,1, \ldots, n\}$ is usually considered to model these linguistic hedges.

For this reason many papers dealing with operations defined on $L_{n}$, usually called discrete operations, have appeared in last years. Dealing with discrete operators, the smoothness condition is usually considered as the discrete counterpart of continuity. In fact, in the discrete framework this property is equivalent to the divisibility property as well as to the Lipschitz condition. Thus, many classes of aggregation functions with some smoothness condition have been studied and characterized. For instance, smooth discrete t-norms and t-conorms were characterized in $[13,14]$, uninorms in $\mathcal{U}_{\min }$ and $\mathcal{U}_{\max }$ and nullnorms in [12], idempotent discrete uninorms in [3], weighted means in [10].

For the case of uninorms, it is well known that there are no smooth uninorms on $L_{n}$ and so, the smoothness condition is only possible in some partial regions of $L_{n}^{2}$, like in the mentioned cases of discrete uninorms in $\mathcal{U}_{\text {min }}$ and $\mathcal{U}_{\text {max }}$ or discrete idempotent uninorms. However, the general case of discrete uninorms having smooth underlying operators has not yet investigated and this is the main goal of this work. The paper is organized as follows. In Section 2 we give some preliminaries that will be used in the paper, and the main results are presented in Section 3. Since smooth t-norms (and t-conorms) are given by the minimum, the Łukasiewicz t-norm (the maximum or the Eukasiewicz t-conorm) or an ordinal sum of these two types of operators, we have divided our results in Section 3 in some subsections devoting a subsection for each possible case of the underlying t-norm and t-conorm of the corresponding uninorm. Section 4 gives general conclusions and future work.

\section{Preliminaries}

We suppose the reader to be familiar with some basic results on uninorms and their classes that can be found for instance in $[4,7,8,11,16]$.

In these preliminaries we recall some known facts on uninorms defined on finite chains, that we will also refer to discrete uninorms. In these cases, the 
concrete scale to be used is not determinant and the only important fact is the number of elements of the scale (see [14]). Thus, given any positive integer $n$, we will deal from now on with the finite chain

$$
L_{n}=\{0,1,2, \ldots, n\} .
$$

We will use indistinctly the interval notation $L_{n}=$ $[0, n]$ and also the usual notations $[0, e]$ and $[e, n]$ when $e \in L_{n}$ for the corresponding subsets of $L_{n}$.

Definition $1 A$ uninorm on $L_{n}$ is a two-place function $U: L_{n}^{2} \rightarrow L_{n}$ which is associative, commutative, increasing in each place and such that there exists some element $e \in L_{n}$, called neutral element, such that $U(e, x)=x$ for all $x \in L_{n}$.

It is clear that the function $U$ becomes a t-norm when $e=n$ and a t-conorm when $e=0$. For any uninorm on $L_{n}$ we have $U(n, 0) \in\{0, n\}$ and a uninorm $U$ is called conjunctive when $U(n, 0)=0$ and disjunctive when $U(n, 0)=n$. The structure of any discrete uninorm $U$ on $L_{n}$ with neutral element $0<e<n$ is always as follows. It is given by a t-norm $T$ on the interval $[0, e]$, by a t-conorm $S$ on the interval $[e, n]$ and it takes values between the minimum and the maximum in all other cases, that is, in the region

$$
A(e)=[0, e] \times[e, n] \cup[e, n] \times[0, e] .
$$

Definition 2 Let $F$ be a binary operator on a finite totally ordered set $L_{n}$. It is said that $F$ verifies the 1-smoothness condition or that $F$ is smooth if whenever $F(i, j)=k$ then

$$
\{F(i-1, j), F(i, j-1)\} \subseteq\{k-1, k\} .
$$

The relation between the Archimedean property $\left(T(x, x)<x\right.$ for all $\left.x \in L_{n} \backslash\{0, n\}\right)$ and the smoothness property for t-norms (and t-conorms) on $L_{n}$ was stated in [13] (see [14] for the current version).

Proposition 1 ([14]) The only Archimedean smooth t-norm and $t$-conorm on $L_{n}$ are, respectively, the Eukasiewicz t-norm

$$
T_{\mathbf{L}}(x, y)=\max (0, x+y-n) \quad \text { for all } x, y \in L_{n},
$$

and the Eukasiewicz t-conorm

$$
S_{\mathbf{L}}(x, y)=\min (n, x+y) \quad \text { for all } x, y \in L_{n} .
$$

Smooth t-norms were characterized in [13] and [14], obtaining that they are the minimum, the Łukasiewicz or an ordinal sum of t-norms of these two classes.

Theorem 1 ([14]) A t-norm $T$ on $L_{n}$ is smooth if and only if there exists a natural number $r$ with $0 \leq r \leq n-1$ and a subset $I$ of $L_{n}, I=\left\{0=a_{0}<\right.$ $\left.a_{1}<\cdots<a_{r}<a_{r+1}=n\right\}$ such that $T$ is given by $T(x, y)=$

$$
\begin{cases}\max \left(a_{i}, x+y-a_{i+1}\right), & \text { if }(x, y) \in\left[a_{i}, a_{i+1}\right]^{2} \\ & \text { and } 0 \leq i \leq r \\ \min (x, y), & \text { otherwise. }\end{cases}
$$

There is a dual result for t-conorms that states that they are the maximum, the Eukasiewicz or ordinal sums of them [14].

Respect to uninorms on $L_{n}$, only the classes of uninorms in $\mathcal{U}_{\text {min }}$ and uninorms in $\mathcal{U}_{\max }$ have been studied and characterized through some partial smoothness conditions in [12]. Specifically, these kinds of uninorms are as follows.

Definition 3 ([12]) A binary operation $U$ : $L_{n}^{2} \longrightarrow L_{n}$ is a uninorm in $\mathcal{U}_{\text {min }}$ with neutral element $0<e<n$ if and only if there is a t-norm $T$ on $[0, e]$ and a t-conorm $S$ on $[e, n]$ such that $U$ is given by

$$
U(x, y)= \begin{cases}T(x, y), & \text { if }(x, y) \in[0, e]^{2}, \\ S(x, y), & \text { if }(x, y) \in[e, n]^{2}, \\ \min (x, y), & \text { elsewhere. }\end{cases}
$$

We will denote a uninorm in $\mathcal{U}_{\min }$ with neutral element $e$ as $U \equiv\langle T, e, S\rangle_{\min }$.

Definition 4 ([12]) A binary operation $U$ : $L_{n}^{2} \longrightarrow L_{n}$ is a uninorm in $\mathcal{U}_{\max }$ with neutral element $0<e<n$ if and only if there is a t-norm $T$ on $[0, e]$ and a t-conorm $S$ on $[e, n]$ such that $U$ is given by

$$
U(x, y)= \begin{cases}T(x, y), & \text { if }(x, y) \in[0, e]^{2}, \\ S(x, y), & \text { if }(x, y) \in[e, n]^{2}, \\ \max (x, y), & \text { elsewhere. }\end{cases}
$$

We will denote a uninorm in $\mathcal{U}_{\max }$ with neutral element e as $U \equiv\langle T, e, S\rangle_{\max }$.

Also idempotent discrete uninorms, that is, those such that $U(x, x)=x$ for all $x \in L_{n}$ were characterized in [3]. For the sake of completeness of the develompment of the present work, we will recall them in Section 3.1, instead of in the current preliminaries. Let just recall here some necessary concepts to give such a characterization.

Definition 5 ([3]) Given any decreasing function $g: L_{n} \rightarrow L_{n}$, we define its completed graph $F_{g}$ as the subset of $\left(L_{n}\right)^{2}$ defined as:

$$
\begin{aligned}
& F_{g}=(\{0\} \times[g(0), n]) \cup(\{n\} \times[0, g(n)]) \\
& \cup\{(x, y) \in[0, n-1] \times[0, n] \mid g(x+1) \leq y \leq g(x)\} .
\end{aligned}
$$

Definition 6 ([3]) A subset $F$ of $\left(L_{n}\right)^{2}$ is said to be Id-symmetrical if for all $(x, y) \in\left(L_{n}\right)^{2}$ it holds that

$$
(x, y) \in F \quad \Longleftrightarrow \quad(y, x) \in F .
$$

The above definition expresses that a subset $F$ of $\left(L_{n}\right)^{2}$ is symmetrical w.r.t. the diagonal $\{(x, x) \mid$ $\left.x \in L_{n}\right\}$. A similar notion of symmetry is introduced for a decreasing function $g: L_{n} \rightarrow L_{n}$.

Definition 7 [3] A decreasing function $g: L_{n} \rightarrow$ $L_{n}$ is said to be Id-symmetrical if its completed graph $F_{g}$ is Id-symmetrical. 


\section{Discrete uninorms with smooth underlying operators}

Let us begin with some general results about discrete uninorms, that will be used in next subsections. Our first result refer to the values of the uninorm in the points $(r, n)$ where $r$ is an idempotent element of $U$ in the interval $] 0, e[$.

Lemma 1 Let $U \equiv\langle T, e, S\rangle$ a uninorm on $L_{n}$. If $r$ is an idempotent element of $U$, with $0<r<e$, then $U(r, n)=r$ or $U(r, n)=n$.

Proof: Consider $U(r, n)=\alpha$, it is known that $r \leq$ $\alpha \leq n$. Now we distinguish two cases:

- If $\alpha \geq e$, then by one side we have $U(\alpha, n)=$ $U(U(r, n), n)=U(r, n)$ and by the other side, by increasingness of $U$ we have $U(\alpha, n) \geq$ $U(e, n)=n$. Therefore, $U(r, n)=n$.

- If $\alpha<e$ then, as $r$ is idempotent of $U$,

$$
\begin{aligned}
r & =U(\alpha, r)=U(U(n, r), r)=U(n, U(r, r)) \\
& =U(n, r),
\end{aligned}
$$

and in this case $U(r, n)=r$.

From the previous lemma, we can deduce a particular case. This situation is depicted in Figure 1 .

Lemma 2 Let $U \equiv\langle T, e, S\rangle$ a uninorm on $L_{n}$. If $r$ is an idempotent element of $U$, with $0<r<e$ and $U(r, n)=r$, then $U(k, m)=\min (k, m)$ for all $(k, m) \in\{(i, j) \mid i \leq r \leq e \leq j$ or $j \leq r \leq e \leq i\}$.

Proof: Let $(k, m)$ be in $\{(i, j) \mid i \leq r \leq e \leq j\}$.

As $U(r, n)=U(r, e)=r$, we have that $U(r, x)=$ $r$ for all $e \leq x \leq n$, and in particular $U(r, m)=r$. Now, using the associativity and commutativity of $U$, we have

$$
\begin{aligned}
k & =U(r, k)=U(U(r, m), k)=U(r, U(m, k))= \\
& =U(r, U(k, m))=U(U(r, k), m)=U(k, m),
\end{aligned}
$$

that concludes the proof.

Analogously to Lemmas 1 and 2, the next results refer to the case when the idempotent element $r$ is in $(e, n)$. This case can be viewed in figure 2 .

Lemma 3 Let $U \equiv\langle T, e, S\rangle$ a uninorm on $L_{n}$. If $r$ is an idempotent element of $U$, with $e<r<n$, then $U(r, 0)=r$ or $U(r, 0)=0$.

Lemma 4 Let $U \equiv\langle T, e, S\rangle$ a uninorm on $L_{n}$. If $r$ is an idempotent element of $U$, with $e<r<n$ and $U(r, 0)=r$, then $U(k, m)=\max (k, m)$ for all $(k, m) \in\{(i, j) \mid i \leq e \leq r \leq j$ or $j \leq e \leq r \leq i\}$.

In view of Theorem 1 and its dual for t-conorms, to classiy all the uninorms with smooth underlying operators, we need to consider different possibilities depending on how is the smooth underlying t-norm and the smooth underlying t-conorm.

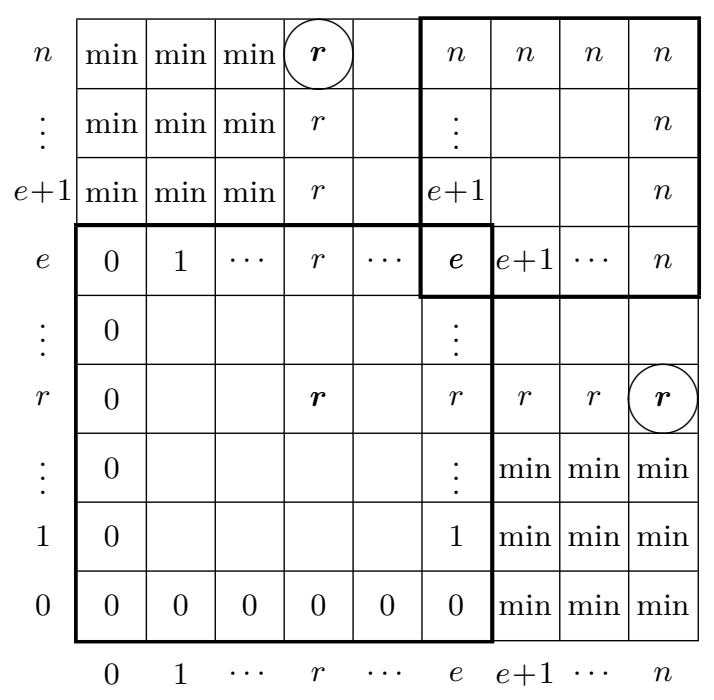

Figure 1: Discrete uninorm with neutral element $e$, idempotent element $0<r<e$ and $U(r, n)=r$.

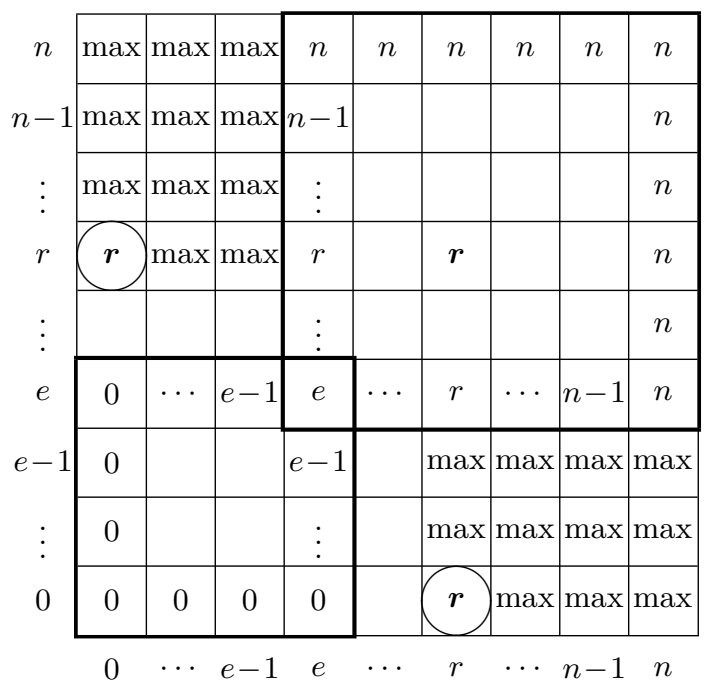

Figure 2: Discrete uninorm with neutral element $e$, idempotent element $e<r<n$ and $U(r, 0)=r$.

\subsection{The idempotent case}

In the case that $T=T_{\mathrm{M}}$ and $S=S_{\mathrm{M}}$, we have that $U$ is an idempotent uninorm. This case was studied in [3], obtaining the following results.

Theorem 2 ([3]) A binary operation $U$ on $L_{n}$ with neutral element $0<e<n$ is an idempotent discrete uninorm if and only if there exists a decreasing function $g:[0, e] \rightarrow[e, n]$ with $g(e)=e$ such that

$U(x, y)= \begin{cases}\min (x, y), & \text { if } y \leq \bar{g}(x) \text { and } x \leq \bar{g}(0) \\ \max (x, y), & \text { elsewhere }\end{cases}$

where $\bar{g}$ is the unique symmetrical extension of $g$, 
given by $\bar{g}(x)=$

$$
\begin{cases}g(x), & \text { if } x \leq e \\ \max \{z \in[0, e] \mid g(z) \geq x\}, & \text { if } e \leq x \leq g(0) \\ 0, & \text { if } x>g(0)\end{cases}
$$

Theorem 3 (i) The number of discrete idempotent uninorms on $L_{n}, n \geq 2$, with neutral element $e \in L_{n}$, is given by

$$
I_{e, n}=\left(\begin{array}{l}
n \\
e
\end{array}\right)
$$

(ii) The total number of discrete idempotent uninorms on $L_{n}, n \geq 2$, is given by

$$
I_{n}=\sum_{e=0}^{n} I_{e, n}=2^{n} .
$$

\subsection{The Eukasiewicz case}

Now we give two more general results referred to the case that the underlying t-norm or the underlying tconorm are Łukasiewicz operators. In the case that $T=T_{\mathbf{L}}$ and the uninorm $U$ is conjunctive, then $U$ must be in the class of $\mathcal{U}_{\min }$.

Proposition 2 Let $U \equiv\langle T, e, S\rangle$ be a conjunctive uninorm on $L_{n}$. If $T=T_{\mathbf{L}}$ then $U \in \mathcal{U}_{\min }$.

Analogously, if $S=S_{\mathbf{L}}$ and $U$ is disjunctive, uninorm $U$ must be in $\mathcal{U}_{\max }$.

Proposition 3 Let $U \equiv\langle T, e, S\rangle$ be a disjunctive uninorm on $L_{n}$. If $S=S_{\mathbf{L}}$ then $U \in \mathcal{U}_{\max }$.

From previous propositions, there will be only two uninorms with underlying operators $T=T_{\mathbf{L}}$ and $S=S_{\mathbf{L}}$. The structure of both uninorms is depicted in Figure 3.

Corollary 1 There are only two uninorms on $L_{n}$, $n \geq 2$ with neutral element $e, 0<e<n$, such that their underlying operators are $T=T_{\mathbf{L}}$ and $S=S_{\mathbf{L}}$ :

- If $U$ is conjunctive, $U \equiv\left\langle T_{\mathbf{L}}, e, S_{\mathbf{L}}\right\rangle_{\min }$.

- If $U$ is disjunctive, $U \equiv\left\langle T_{\mathbf{L}}, e, S_{\mathbf{L}}\right\rangle_{\max }$.

From this result we can calculate the number of uninorms with Łukasiewicz underlying operators varying the neutral element in $L_{n}$ (including the cases $e=0$, the Łukasiewicz t-conorm and $e=1$, the Łukasiewicz t-norm).

Corollary 2 The total number of uninorms on $L_{n}$, $n \geq 1$, such that their underlying operators are $T=$ $T_{\mathbf{L}}$ and $S=S_{\mathbf{L}}$ is $2 n$.
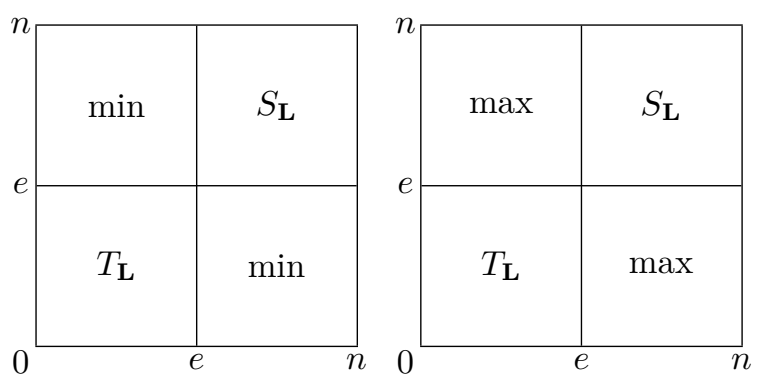

Figure 3: Discrete uninorms with underlying operators $T=T_{\mathbf{L}}$ and $S=S_{\mathbf{L}}$, conjunctive (left) and disjunctive (right).

\subsection{Case $T=T_{\mathrm{M}}$ and $S=S_{\mathrm{L}}$}

In this subsection we will deal with uninorms such that their underlying operators are $T=T_{\mathbf{M}}$ and $S=S_{\mathbf{L}}$.

Note that, from the previous case, if the uninorm is disjunctive and $S=S_{\mathbf{L}}$, then it must be in $\mathcal{U}_{\max }$. Considering that there will always exists $r \in L_{n}$ such that $0 \leq r \leq e$, and $U(r, n)=n$, the uninorm $U$ restricted to $[r, n]^{2}$ satisfies Proposition 3 . From this and Lemma 2, we can deduce the general structure of the uninorms with $T=T_{\mathbf{M}}$ and $S=S_{\mathbf{L}}$. This structure can be observed in Figure 4.

Proposition 4 Let $U \equiv\langle T, e, S\rangle$ be a uninorm on $L_{n}$ such that $T=T_{\mathbf{M}}$ and $S=S_{\mathbf{L}}$. Let $0 \leq r \leq e$ be the smallest element such that $U(r, n)=\max (r, n)=n$. Then $U$ is given by

$U(x, y)= \begin{cases}\min (x, y), & \text { if }(x, y) \in[0, e]^{2}, \\ S_{\mathbf{L}}(x, y), & \text { if }(x, y) \in[e, 1]^{2}, \\ \max (x, y), & \text { if }(x, y) \in[r, e] \times[e, n] \text { or } \\ & (x, y) \in[e, n] \times[r, e], \\ \min (x, y), & \text { otherwise. }\end{cases}$

Again, with this result is easy to count the total number of uninorms in this case.

Corollary 3 (i) The number of uninorms $U \equiv$ $\langle T, e, S\rangle$ on $L_{n}, n \geq 2$ with neutral element $e \in L_{n}$ such that their underlying operators are $T=T_{\mathbf{M}}$ and $S=S_{\mathbf{L}}$ is given by

$$
M L_{e, n}=e+1 \text {. }
$$

(ii) The total number of uninorms on $L_{n}, n \geq 1$, such that their underlying operators are $\bar{T}=$ $T_{\mathbf{M}}$ and $S=S_{\mathbf{L}}$ is

$$
M L_{n}=\sum_{e=0}^{n} M L_{e, n}=\frac{n(n+1)}{2}+1 .
$$

3.4. Case $T=T_{\mathbf{L}}$ and $S=S_{\mathbf{M}}$

Now we study the case of uninorms such that their underlying operators are $T=T_{\mathbf{L}}$ and $S=S_{\mathbf{M}}$. It 


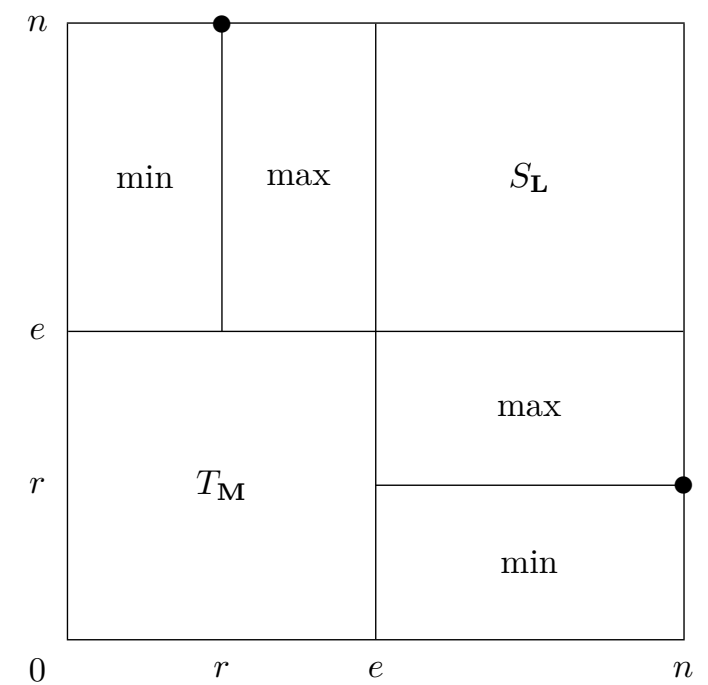

Figure 4: Structure of a discrete uninorm with underlying operators $T=T_{\mathbf{M}}$ and $S=S_{\mathbf{L}}$.

is very similar to the previous one, and it can be proved using duality. The structure of such uninorms can be viewed in Figure 5

Proposition 5 Let $U \equiv\langle T, e, S\rangle$ such that $T=T_{\mathbf{L}}$ and $S=S_{\mathrm{M}}$. Let $e \leq r \leq n$ be the biggest element such that $U(0, r)=\min (0, r)=0$. Then $U$ is given by

$U(x, y)= \begin{cases}T_{\mathbf{L}}(x, y), & \text { if }(x, y) \in[0, e]^{2}, \\ \max (x, y), & \text { if }(x, y) \in[e, 1]^{2}, \\ \min (x, y), & \text { if }(x, y) \in[0, e] \times[e, r] \text { or } \\ & (x, y) \in[e, r] \times[0, e] \\ \max (x, y), & \text { otherwise. }\end{cases}$

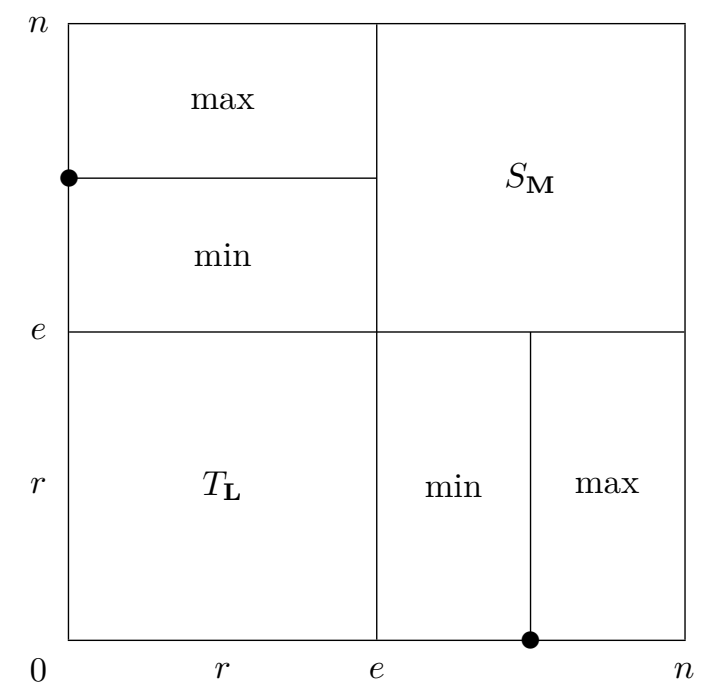

Figure 5: Structure of a discrete uninorm with underlying operators $T=T_{\mathbf{L}}$ and $S=S_{\mathbf{M}}$.

In the next corollary we give the total number of uninorms in this case.

\begin{tabular}{|c|c|c|}
\hline $\mathrm{T}$ & $S_{\mathbf{M}}$ & $S_{\mathbf{L}}$ \\
\hline$T_{\mathbf{M}}$ & $\left(\begin{array}{l}n \\
e\end{array}\right)$ & $e+1$ \\
\hline$T_{\mathbf{L}}$ & $n-e+1$ & 2 \\
\hline
\end{tabular}

Table 1: Number of discrete uninorms $U \equiv\langle T, e, S\rangle$ with neutral element $e \in L_{n}, 0<e<n$, depending on which are their underlying operators.

\begin{tabular}{|c|c|c|}
\hline$T^{T}$ & $S_{\mathbf{M}}$ & $S_{\mathbf{L}}$ \\
\hline$T_{\mathbf{M}}$ & $2^{n}$ & $\frac{n(n+1)}{2}+1$ \\
\hline$T_{\mathbf{L}}$ & $\frac{n(n+1)}{2}+1$ & $2 n$ \\
\hline
\end{tabular}

Table 2: Total number of discrete uninorms $U \equiv$ $\langle T, e, S\rangle$ on $L_{n}$, with $T$ and $S$ idempotent or Archimedean.

Corollary 4 (i) The number of uninorms $U \equiv$ $\langle T, e, S\rangle$ on $L_{n}, n \geq 2$ with neutral element $e \in L_{n}$ such that their underlying operators are $T=T_{\mathbf{L}}$ and $S=S_{\mathbf{M}}$ is given by

$$
L M_{e, n}=n-e+1 \text {. }
$$

(ii) The total number of uninorms on $L_{n}, n \geq 1$, sucht that their underlying operators are $T=$ $T_{\mathbf{L}}$ and $S=S_{\mathbf{M}}$ is

$$
L M_{n}=\sum_{e=0}^{n} L M_{e, n}=\frac{n(n+1)}{2}+1 .
$$

So, if we join all the results above, we can obtain the number of uninorms in any of the considered cases which is represented in tables 1 (with a fixed neutral element $e$ ) and 2 (in general).

Remark 1 Note that in such a table, there are some cases counted twice. For instance, $T=T_{\mathbf{L}}$ is included in both the cases: $T=T_{\mathbf{L}}$ and $S=S_{\mathbf{L}}$, and $T=T_{\mathbf{L}}$ and $S=S_{\mathbf{M}}$.

In view of the previous remark, we obtain the following proposition, which gives the total number of uninorms considered in this section.

Proposition 6 The total number of discrete uninorms on $L_{n}, n \geq 1$, such that their underlying operators are idempotent or Archimedean is given by

$$
2^{n}+n(n+3)-2 .
$$

\subsection{General case: when $T$ or $S$ are ordinal sums}

After studying all cases for $T$ and $S$ being idempotent or Archimedean operators, the remaining case 
is whenever $T$ and/or $S$ are ordinal sums. To start with this case, we give a similar result to Proposition 2 .

Proposition 7 Let $U \equiv\langle T, e, S\rangle$ be a uninorm such that $T$ is smooth and there exist two consecutive idempotent elements of $U, r, s \in L_{n}, 0<r<$ $s<e$ with $s \geq r+2$. If $U(r, n)=r$, then $U(k, m)=$ $\min (k, m)$ for all $(k, m) \in[r, s[\times[e, n] \cup[e, n] \times[r, s[$.

By duality, we have a similar result to Proposition 3 for uninorms with idempotent elements greater than the neutral element $e$ of $U$.

Proposition 8 Let $U \equiv\langle T, e, S\rangle$ be a uninorm such that $S$ is smooth and there exist two consecutive idempotent elements of $U, r, s \in L_{n}, e<r<$ $s<n$ with $s \geq r+2$. If $U(s, 0)=s$, then $U(k, m)=$ $\max (k, m)$ for all $(k, m) \in] r, s] \times[0, e] \cup[0, e] \times] r, s]$.

Now we present construction example for uninorms $U \equiv\langle T, e, S\rangle$ with $T$ and $S$ smooth operators, in which $T$ is an ordinal sum and $S$ is Łukasiewicz.

Example 1 Consider $L_{7}$, we want to determine all possible uninorms with neutral element $e=4$, underlying smooth operators, and idempotent elements $I=\{0,2,4,7\}$, that is, $S=S_{\mathbf{L}}$ and $T$ is an ordinal sum with one non-trivial idempotent element. This situation can be observed in figure 6. Empty squares correspond to not-known values of $U$. Now, we will distinguish some cases:

- If $U$ is disjunctive, using Proposition 3, we have that $U \in \mathcal{U}_{\max }$.

- If $U$ is conjunctive. By Proposition 7, $U(i, j)=$ $\min (i, j)$ for all $(i, j) \in[0,2) \times[5,7] \cup[5,7] \times$ $[0,2)$. Now, depending on the values of $U(2,7)$ we have the following two subcases:

- If $U(2,7)=2$, if we consider uninorm $U$ on the set $[2,7]^{2}$, it's a conjunctive uninorm $\left.U\right|_{[2,7]}$, and thus, by Theorem 2, this uninorm has to be in $\mathcal{U}_{\min }$. Then, adding all this information, $U \in \mathcal{U}_{\text {min }}$.

- If $U(2,7)=7$, we can take the operator $\left.U\right|_{[2,7]}$, that it is disjunctive, with both underlying Eukasiewicz operators and therefore $\left.U\right|_{[2,7]} \in \mathcal{U}_{\max }$.

Then, there are only three uninorms with neutral element $e=4$, underlying smooth operators, and idempotent elements $I=\{0,2,4,7\}$. This three uninorms can be viewed in Figure 7.

In view of this example, if we know the idempotent elements of the uninorm, by using Propositions 7 and 8 , we could construct all the uninorms with underlying operators $T$ and $S$ smooth.

Note that in all the studied cases it appears that an Id-symmetrical decreasing function $g$ on $L_{n}$ with fixed point $e$ exists, such that the uninorm in the region $A(e)$ is given by the minimum under the graph

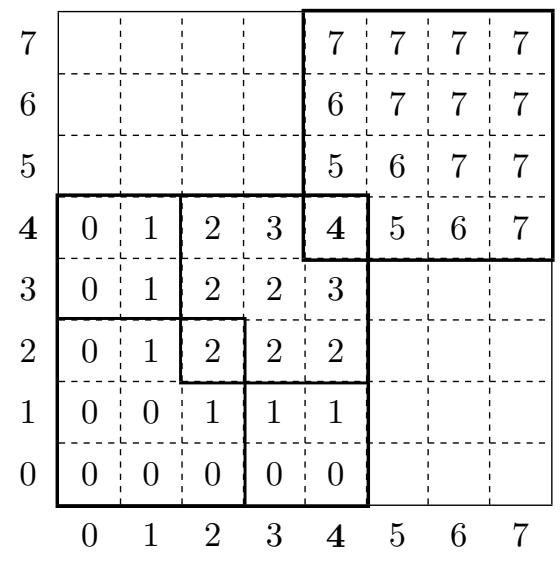

Figure 6: Structure of a discrete uninorm in $L_{7}$ with neutral element 4 , smooth underlying operators and idempotent elements $\{0,2,4,7\}$.

of $g$ and by the maximum over this graph. The only restriction of $g$ is that it must be constant in any interval where the underlying t-norm $T$ (or the underlying t-conorm $S$ ) is given by a tukasiewicz summand. To formalize this idea and to used it in characterizing all discrete uninorms with smooth underlying operations is part of our future work on this topic.

\section{Conclusions and future work}

In this work the structure of uninorms $U \equiv\langle T, e, S\rangle$ defined on a finite chain $L_{n}$, when the underlying operators $T$ and $S$ are smooth has been studied, obtaining a characterization of the cases when these operations are idempotent or Archimedean. Also, the number of operations with a fixed neutral element $e \in L_{n}$ has been found as well as the total number of uninorms on $L_{n}$ such that its underlying operators are smooth. For the case that $T$ or $S$ are ordinal sums, a construction example has been given, offering all possible uninorms with this configuration of underlying smooth operators.

In a future work, our goal will be to establish a general characterization of all possible uninorms with smooth underlying operations, and to compute the total number of these uninorms.

\section{Acknowledgments.}

This work has been supported by the Spanish grant MTM2009-10320, with FEDER support.

\section{References}

[1] B. De Baets, Idempotent uninorms, European Journal of Operational Research, 118:631-642, 1999. 
[2] B. De Baets and J. Fodor, A single-point characterization of representable uninorms, Fuzzy Sets and Systems, 202:89-99, 2012.

[3] B. De Baets, J. Fodor, D. Ruiz-Aguilera and J. Torrens, Idempotent Uninorms on Finite Ordinal Scales, International Journal of Uncertainty, Fuzziness and Knowledge-Based Systems, 17:114, World Scientific, 2009.

[4] P. Drygaś, Discussion of the structure of uninorms, Kybernetika, 41:213-226, 2005.

[5] P. Drygaś, On the structure of continuous uninorms, Kybernetika, 43:183-196, 2007.

[6] J.C. Fodor, B. De Baets and T. Calvo, Characterization of uninorms with given continuous underlying t-norms and $t$-conorms, submitted.

[7] J. Fodor, R. Yager and A. Rybalov, Structure of uninorms, International Journal of Uncertainty, Fuzziness and Knowledge-based Systems, 5:411427, 1997.

[8] S. Hu and Z. Li, The structure of continuous uni-norms, Fuzzy Sets and Systems, 124:43-52, 2001.

[9] E. P. Klement, R. Mesiar, and E. Pap, Triangular norms. Kluwer, Dordrecht, 2000.

[10] A. Kolesarova, G. Mayor and R. Mesiar, Weighted ordinal means, Information Sciences, 177 (2007) 3822-3830.

[11] J. Martín, G. Mayor and J. Torrens, On locally internal monotonic operations, Fuzzy Sets and Systems, 137:27-42, 2003.

[12] M. Mas, G. Mayor and J. Torrens, $t$-Operators and uninorms on a finite totally ordered set, International Journal of Intelligent Systems, 14:909-922, 1999.

[13] G. Mayor and J. Torrens, On a class of operators for expert systems, International Journal of Intelligent Systems, 8:771-778, 1993.

[14] G. Mayor and J. Torrens, Triangular norms in discrete settings. In E.P. Klement and R. Mesiar, editors, Logical, Algebraic, Analytic, and Probabilistic Aspects of Triangular Norms, pages 189-230, Elsevier, Amsterdam, 2005.

[15] D. Ruiz and J. Torrens, Distributivity and conditional distributivity of a uninorm and a continuous $t$-conorm, IEEE Transactions on Fuzzy Systems, 14:180-190, 2006.

[16] D. Ruiz-Aguilera, J. Torrens, B. De Baets and J. Fodor, Some Remarks on the Characterization of Idempotent Uninorms. In E. Hüllermeier, R. Kruse and F. Hoffmann,editors, proceedings of the $13^{\text {th }}$ IPMU Conference 2010, Lecture Notes in Computer Science 6178, pages 425-434, Springer-Verlag, 2010.

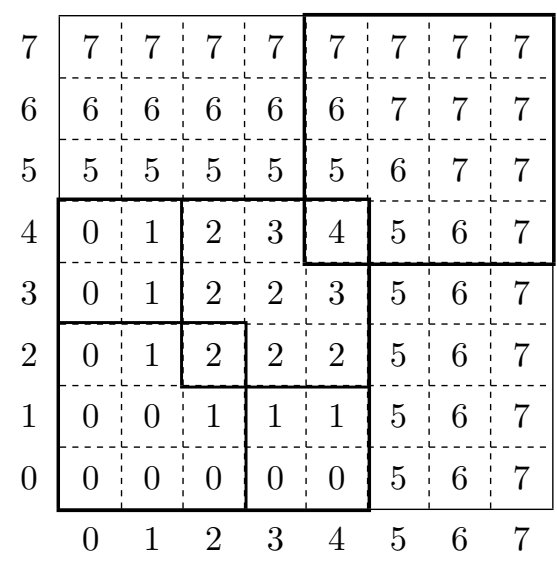

\begin{tabular}{l|c:c:c:c|c:c:c:c|}
\hline 7 & 0 & 1 & 2 & 3 & 7 & 7 & 7 & 7 \\
\hline 6 & 0 & 1 & 2 & 3 & 6 & 7 & 7 & 7 \\
\hline 5 & 0 & 1 & 2 & 3 & 5 & 6 & 7 & 7 \\
\cline { 2 - 11 } & 0 & 1 & 2 & 3 & 4 & 5 & 6 & 7 \\
\hline 3 & 0 & 1 & 2 & 2 & 3 & 3 & 3 & 3 \\
\hline & 0 & 1 & 2 & 2 & 2 & 2 & 2 & 2 \\
\hdashline & 0 & 0 & 1 & 1 & 1 & 1 & 1 & 1 \\
0 & 0 & 0 & 0 & 0 & 0 & 0 & 0 & 0 \\
\hline
\end{tabular}

\begin{tabular}{l|c|c|c|c|c|c|c|c|}
\hline 7 & 0 & 1 & 7 & 7 & 7 & 7 & 7 & 7 \\
\hline 6 & 0 & 1 & 6 & 6 & 6 & 7 & 7 & 7 \\
5 & 0 & 1 & 5 & 5 & 5 & 6 & 7 & 7 \\
4 & 0 & 1 & 2 & 3 & 4 & 5 & 6 & 7 \\
\hline 3 & 0 & 1 & 2 & 2 & 3 & 5 & 6 & 7 \\
\hline 2 & 0 & 1 & 2 & 2 & 2 & 5 & 6 & 7 \\
\hline 1 & 0 & 0 & 1 & 1 & 1 & 1 & 1 & 1 \\
\hdashline 0 & 0 & 0 & 0 & 0 & 0 & 0 & 0 \\
\hline
\end{tabular}

Figure 7: The only three uninorms in $L_{7}$ with neutral element 4, smooth underlying operators and idempotent elements $\{0,2,4,7\}$. From top to bottom, the uninorm in $\mathcal{U}_{\max }$, in $\mathcal{U}_{\text {min }}$ and a uninorm that is not in $\mathcal{U}_{\max }$ nor $\mathcal{U}_{\text {min }}$. 